\title{
The Potential Impact of Self-medication and Drug Misuse Practice Among Youth Population in Kinshasa, Democratic Republic of Congo
}

\author{
Kalonji Katengele ${ }^{1}$, Fumwakwau Kiniati ${ }^{2}$, Nkanga Isalomboto ${ }^{1}$, Didi Kialengila Mana ${ }^{1}$, * \\ ${ }^{1}$ Faculty of Medicine and Pharmaceutical Sciences, Laboratory for Medicines Analysis and Health Products, Kinshasa XI, Democratic \\ Republic of Congo \\ ${ }^{2}$ Coordination Center of Research and Documentation in the Social Sciences Serving Sub-saharan Africa, University of Kinshasa, Kinshasa, \\ Democratic Republic of Congo
}

Email address:

manakialengila@gmail.com (D. K. Mana)

${ }^{*}$ Corresponding author

\section{To cite this article:}

Kalonji Katengele, Fumwakwau Kiniati, Nkanga Isalomboto, Didi Kialengila Mana. The Potential Impact of Self-medication and Drug Misuse Practice Among Youth Population in Kinshasa, Democratic Republic of Congo. American Journal of Biomedical and Life Sciences. Vol. 9, No. 1, 2021, pp. 69-77. doi: 10.11648/j.ajbls.20210901.19

Received: January 29, 2021; Accepted: February 6, 2021; Published: February 23, 2021

\begin{abstract}
Self-medication refers to the act of medicating oneself with no guidance from a physician. There are sevral factors that promote self-medication along with drug misuse among the population. In the frame of developing countries, such as the Democratic Republic of Congo (DRC), these factors include lack of enforcement of laws governing the pharmaceutical sector, poor mindset of the population as well as easy access to drugs without official medical prescriptions. The purpose of this study was to evaluate the extent to which self-medication and drug misuse practices affect the youth Congolese population health in the DRC's capital city, Kinshasa. Three sectors of Kinshasa were selected for a cross-sectional study conducted for five months (January to Mai 2020). Pharmacists or their auxiliairies were asked to draw up the list of ten drugs often purchased without medical prescriptions. In each of the selected pharmacies, 3 patients using self-medication were interviewed. A total of 120 phrarmacists or their auxiliairies and 360 patients (180 men and 180 women) were selected using systematic random sampling technique. Of the 120 questionnaires completed, a list of ten most used drugs in self-medication was established in the following dicreasing quantitative order: 106, 85, 83, 66, 66, 58, 51, 38, 29 and 23 pharmacists or their auxiliairies cited Amoxicillin capsule, Emergency Contraceptive Pill tablet, Paracetamol tablet, Dexamethasone tablet, Cyproheptadine tablet, Tetracyclin capsule, Levamisole tablet, Albendazole tablet, Quinine and Diclofenac injectables, respectively. Out of 360 questionnaires distributed, all medicines were administered by the route recommended by the physician except Dexamethasone tablet, Cyproheptadine tablet and Diclofenac injection where in 30.1\%, 38.1\% and 89.7\% of selfmedication cases the route of administration was other than the recommended route. From the recorded data, the age of most patients varied between 15-24 years old. Girls were more in self-medication than boys. Sometimes, the disease for which the drug was used, as well as the dosing, were found to be different from the instructions in patients' leaflet. And lastly, for most patients, the main source of information was family and/or friends. This study demonstrated that the practice of self-medication is very widespread in Kinshasa, unfortunately even antibiotics are misused, exposing the population to high risk of antimicrobial resistance development. The present findings encourage further investigations at national level to better advise the dicision makers to take appropriate measures to substantially mitigate self-medication and drug misuse practices.
\end{abstract}

Keywords: Self-medication, Drug Misuse, Antimicribial Resistance, Drug Toxicity, Kinshasa/Democratic Republic of Congo (DRC)

\section{Introduction}

Self-medication is defined as the intake of selected drugs for treating self-recognized or self-diagnosed illness or symptoms without medical evaluation. Self-medication constitues a worldwide health issue [1]. It remains a common 
practice in Democratic Republic of Congo (DRC). Several factors are at the root of this practice, including the lack of enforcement of laws governing the dispensing of drugs, the poverty of the population, the absence of pharmacist in pharmacies and the extended waiting time required for medical consultations in the public healthcare system.

Rational self-medication can be beneficial to the society, in the sense that it allows people to get involved in recovering their own health, learning about the use of drugs and saving time [1]. Despite this possible positive impact, self-medication remains a dangerous practice because drugs are not supposed to be consumed as goods, and their management should not be left in hands of nonexperts.

Potential danger of irrational self-medication include: dangerous drug interactions, misuse of antibiotics leading to the emergence of antibiotic resistant strains, severe side effects, waste of time and money to relieve pain, increase health care and morbi-mortality rate [2].

The lack of enforcement of laws governing the pharmaceutical sectors has promoted not only the generalization of self-medication practice but also the misuse of easily obtained drugs. Several previous studies conducted in DRC reported the misuse of certain drugs taken individually. Among these, we can cite for example studies conducted on Cyproheptadine, Emergency contraceptive pill (ECP), Dexamethasone tablets and Amoxicillin capsule [2-6]. To our best knowledge, no study has focused on a list of drugs often used through self-medication among youth population in Kinshasa.

It is in this context that this survey was initiated in order to assess the extent to which self-medication and drug misuse occured among youth population in three town's sectors of Kinshasa. This was done in order to establish the profile of drugs often purchased without prescription, to determine the risks of this practice among youth and as far as possible, provide concrete proposals to the regulatory authorities to help them ensure the population safety.

\section{Methodology}

\subsection{Study Site and Sampling Method}

A cross-sectional questionnaire-based study was carried out from January to Mai 2020 in three town's sectors of Kinshasa (Lemba, Matete and Limete townships). Those townships were selected because of their proximity to university of Kinshasa and their life styles (population behavior to the up-to-date fashion) [4]. The first stage was the selection of fourty community pharmacies by townships using a simple random sampling technique. The second stage consisted of enrolment of three patients using self-medication practice in each selected community pharmacy. A total of 480 questionnaires were distributed, 120 to pharmacists or sale persons working in the drugstores and 360 questionnaires to their patients using selfmedication practice. Patients using self-medication were identified after discussing with the investigator.

After the facilitator explained how to fill in the questionnaire, pharmacists or their auxiliairies were asked to list up ten drugs often purchased without medical prescriptions. And patients were interviewed on their self-medication practice in conjunction with the following: the routes of administration, the dosages used, the effect sought, the sex and the age of the patients as well as their information source.

\subsection{Ethical Issues}

The study protocol was assessed and approved by the Internal Review Board of the University of Kinshasa, Faculty of Pharmaceutical Sciences (protocol approval number 161/CNES/BN/PNMF/2020). According to the Helsinki declaration II, a written informed consent was signed prior to data collection. A detailed explanation on the goal of the study was given and the confidentiality guaranteed.

\subsection{Data Collection and Analysis}

A face-to-face interview using a structured questionnaire was used to collect the information from pharmacists or thier auxilairies and from patients. The infomation regarding the most used medication without medical prescription was collected. The route of administration, the objective for which the medication is used, the dosage used, and the information source were recorded. The age and the sex of the person involved in self-medication practice were noted. The collected data were analyzed using the statistical package for social sciences (SPSS) of the University of Kinshasa version 20.0 and the results were presented using percentages and absolute tablet. To investigate the possible relations between sex, age and the medicine used by self-medication, Chi-square test was performed. A p-value of $<$ 0.05 was considered statistically significant.

Table 1. Frequency of the drugs commonly used by self-medication.

\begin{tabular}{lll}
\hline Products & Frenquency & Percentage \\
\hline Amoxicilline capsule & 106 & 88.3 \\
Emergency pill tablet & 85 & 70.8 \\
Paracétamol tablet & 83 & 69.2 \\
Dexamethasone tablet & 66 & 55 \\
Cyproheptadine tablet & 66 & 55 \\
Tetracycline capsule & 58 & 48.3 \\
Levamisole tablet & 51 & 42.5 \\
Albendazole tablet & 38 & 31.6 \\
Quinine injectable & 29 & 24.1 \\
Diclofenac injectable & 23 & 19.1 \\
\hline
\end{tabular}

\section{Results}

\subsection{Drugs Commonly Used by Self-medication}

From the 120 questionnaires completed by pharmacists or their auxilairies, a list of ten most purchased medicine without medical prescription was established (Table 1).

\subsection{Socio-demographic Characteristics Among the Patients}

Out of 360 questionnaires distributed, 180 men and 180 women responded, 181 were between 15-24 years old and 179 were $\geq 25$ years old. According to the data, variable proportions of patients confirmed to have practiced selfmedication with different drugs: Amoxicillin 326 (90.6\%), 
Emergency Contraceptive Pill (ECP) 180 (100\% of women), Paracetamol 303 (84.1\%), Dexamethasone 236 (65.6\%), Cyproheptadine, 236 (65.6\%), Tetracyclin 203 (56.4\%), Levamisole 191 (53.1\%), Albendazole 180 (50.0\%), Quinine 171 (47.5\%) and Diclofenac 155 (43.1\%).

The age of most patients varied between 15-24 years old. And lastly, in terms of gender, female were in the majority involved in non-prescription practice for the following medicines: ECP, Dexamethasone, Cyproheptadine, Levamisole, Albendazole tablets, Quinine and Diclofenac injectables. Whereas, males were more involved in selfmedication with Amoxycillin, Tetracycline and Paracetamol as clearly described in Tables 2 and 3 .

Self medication practice and drug misuse were signifantly associated with sex; females used more dexamethasone tablet (97.2\%), cyproheptadine tablet (94.4\%), levamisole tablet (91.7\%), albendazole tablet (78.9\%), quinine injectable
(90.6\%) and diclofenac injectable $(82.8 \%)$ than men, dexamethasone tablet (33.9\%), cyproheptadine tablet $(36.7 \%)$, levamisole tablet $(14.4 \%)$, albendazole tablet $(21.1 \%)$, quinine injectable $(4.4 \%)$ and diclofenac injectable (3.3\%) (All p-values were $<0.001$ ) while men used more amoxicilline capsule (100\%), paracetamol table (94.4\%) and tetracycline capsule $(63.3 \%)$ than females, amoxicilline capsule $(81.1 \%)$, paracetamol table $(49.4 \%)$ and tetracycline capsule $(73.9 \%)$ (All p-values were $<0.008)$ (Table 2$)$. This practice was also significantly associated with age (patients aged 15 - 25) used more paracetamol tablet (100\%), cyproheptadine tablet $(77.9 \%)$ and albendazole tablet $(58 \%)$ than those aged $\geq 25$, paracetamol tablet $(68.2 \%)$, cyproheptadine tablet $(53.1 \%)$ and albendazole table $(41.9 \%)$ (all p-value were $<0.002$ ) whereas patients aged $\geq 25$ used more ECP $(59.2 \%)$ and tetracycline $(68.2 \%)$ than those aged $15-24$, ECP (41.4\%) and tetracycline (68.2\%) (Table 3).

Table 2. Socio-demographic characteristics of persons of interest and attitude to self medication practice based on the sex $(n=360)$.

\begin{tabular}{|c|c|c|c|c|c|c|}
\hline \multirow{2}{*}{ Variables } & \multicolumn{2}{|c|}{ Amoxicilline capsule } & \multirow{2}{*}{ Khi2/p } & \multicolumn{2}{|c|}{ Tetracycline capsule } & \multirow{2}{*}{ Khi2/p } \\
\hline & Users & Non users & & users & Non users & \\
\hline \multicolumn{7}{|l|}{ Sex } \\
\hline Male & $180 / 100$ & $0 / 0$ & \multirow{3}{*}{$14.566 /<0.001$} & $114 / 63.3$ & $66 / 36.7$ & \multirow[t]{3}{*}{$7.0597 / 0.008$} \\
\hline Female & $146 / 81.1$ & $34 / 18.9$ & & $89 / 49.4$ & $91 / 50.6$ & \\
\hline Total & $326 / 90.6$ & $34 / 9.4$ & & $203 / 56.4$ & $157 / 43.6$ & \\
\hline \multirow{2}{*}{ Variables } & \multicolumn{2}{|c|}{ ECP tablet } & \multirow{2}{*}{ Khi2/p } & \multicolumn{2}{|c|}{ Levamisole tablet } & \multirow{2}{*}{ Khi2/p } \\
\hline & Users & Non users & & users & Non users & \\
\hline \multicolumn{7}{|l|}{ Sex } \\
\hline Male & $0 / 0$ & $180 / 100$ & \multirow{3}{*}{$10.0000 / 0.002$} & $26 / 14.4$ & $154 / 85.6$ & \multirow{3}{*}{$\begin{array}{l}215.483 / \\
<0.001\end{array}$} \\
\hline Female & $180 / 100$ & $0 / 0$ & & $165 / 91.7$ & $15 / 8.3$ & \\
\hline Total & $180 / 50$ & $180 / 50$ & & $191 / 53.1$ & $169 / 46.9$ & \\
\hline \multirow{2}{*}{ Variables } & \multicolumn{2}{|c|}{ Paracétamol tablet } & \multirow{2}{*}{ Khi2/p } & \multicolumn{2}{|c|}{ Albendazole tablet } & \multirow{2}{*}{ Khi2/p } \\
\hline & Users & Non users & & Users & Non users & \\
\hline \multicolumn{7}{|l|}{ Sex } \\
\hline Male & $170 / 94.4$ & $10 / 5.6$ & \multirow{3}{*}{$28.536 /<0.001$} & $38 / 21.1$ & $142 / 78.9$ & \multirow[t]{3}{*}{$120.178 / 0.001$} \\
\hline Female & $133 / 73.9$ & $47 / 26.1$ & & $142 / 78.9$ & $38 / 21.1$ & \\
\hline Total & $303 / 84.2$ & $57 / 15.8$ & & $180 / 50$ & $180 / 50$ & \\
\hline \multirow{2}{*}{ Variables } & \multicolumn{2}{|c|}{ Dexamethasone tablet } & \multirow{2}{*}{ Khi2/p } & \multicolumn{2}{|c|}{ Quinine injectable } & \multirow{2}{*}{ Khi2/p } \\
\hline & Users & Non users & & users & Non users & \\
\hline \multicolumn{7}{|l|}{ Sex } \\
\hline Male & $61 / 33.9$ & $119 / 66.1$ & \multirow{3}{*}{$159.874<0.001$} & $8 / 4.4$ & $172 / 95.6$ & 267.614/ \\
\hline Female & $175 / 97.2$ & $5 / 2.8$ & & $163 / 90.6$ & $17 / 9.4$ & $<0.001$ \\
\hline Total & $236 / 65.6$ & $124 / 34.4$ & & $171 / 47.5$ & $189 / 52.5$ & \\
\hline Voriol & Cyprohe & bblet & & Diclofen: & ble & \\
\hline Variables & Users & Non users & Khi2/p & users & Non users & Khi2/p \\
\hline Sex & & & & & & \\
\hline Male & $66 / 36.7$ & $114 / 63.3$ & $133056 /<0001$ & $6 / 3.3$ & $174 / 96.7$ & $231.680 /$ \\
\hline Female & $170 / 94.4$ & $10 / 5.6$ & $133.056 /<0.001$ & $149 / 82.8$ & $31 / 17.2$ & $<0.001$ \\
\hline Total & $236 / 65.6$ & $124 / 34.4$ & & $155 / 43.1$ & $205 / 56.9$ & \\
\hline
\end{tabular}

Table 3. Socio-demographic characteristics of persons of interest and attitude to self medication practice based on the age $(n=360)$.

\begin{tabular}{|c|c|c|c|c|c|c|}
\hline \multirow{2}{*}{ Variables } & \multicolumn{2}{|c|}{ Amoxicilline capsule } & \multirow{2}{*}{ Khi2/p } & \multicolumn{2}{|c|}{ Tetracycline capsule } & \multirow{2}{*}{ Khi2/p } \\
\hline & Users & Non users & & users & Non users & \\
\hline Age (years) & & & & & & $20.0474 /$ \\
\hline $15-24$ & $166 / 91.7$ & $15 / 8.3$ & $0.569 / 0.450$ & $81 / 44.8$ & $100 / 55.2$ & $<0.001$ \\
\hline$\geq 25$ & $160 / 89.4$ & $19 / 10.6$ & & $122 / 68.2$ & $57 / 31.8$ & \\
\hline Total & $326 / 90.6$ & $34 / 9.4$ & & $203 / 56.4$ & $157 / 43.6$ & \\
\hline
\end{tabular}




\begin{tabular}{|c|c|c|c|c|c|c|}
\hline \multirow{2}{*}{ Variables } & \multicolumn{2}{|c|}{ ECP tablet } & \multirow{2}{*}{ Khi2/p } & \multicolumn{2}{|c|}{ Levamisole tablet } & \multirow{2}{*}{ Khi2/p } \\
\hline & Users & Non users & & users & Non users & \\
\hline \multicolumn{7}{|l|}{ Age (years) } \\
\hline $15-24$ & $75 / 41.4$ & $106 / 58.6$ & \multirow{3}{*}{$11.382 /<0.001$} & $101 / 55.8$ & $80 / 44.2$ & \multirow{3}{*}{$1.102 / 0.294$} \\
\hline$\geq 25$ & $106 / 59.2$ & $73 / 40.8$ & & $90 / 50.3$ & $89 / 49.7$ & \\
\hline Total & $180 / 50$ & $180 / 50$ & & $191 / 53.1$ & $169 / 46.9$ & \\
\hline \multirow{2}{*}{ Variables } & \multicolumn{2}{|c|}{ Paracétamol tablet } & \multirow{2}{*}{ Khi2/p } & \multicolumn{2}{|c|}{ Albendazole tablet } & \multirow{2}{*}{ Khi2/p } \\
\hline & Users & Non users & & Users & Non users & \\
\hline \multicolumn{7}{|l|}{ Age (years) } \\
\hline $15-24$ & $181 / 100$ & $0 / 0$ & \multirow{3}{*}{$68.479 /<0.001$} & $105 / 58$ & $76 / 42$ & \multirow{3}{*}{$9.345 / 0.002$} \\
\hline$\geq 25$ & $122 / 68.2$ & $57 / 31.8$ & & $75 / 41.9$ & $104 / 58.1$ & \\
\hline Total & $303 / 84.2$ & $57 / 15.8$ & & $180 / 50$ & $180 / 50$ & \\
\hline \multirow{2}{*}{ Variables } & \multicolumn{2}{|c|}{ Dexamethasone tablet } & \multirow{2}{*}{ Khi2/p } & \multicolumn{2}{|c|}{ Quinine injectable } & \multirow{2}{*}{ Khi2/p } \\
\hline & Users & Non users & & users & Non users & \\
\hline \multicolumn{7}{|l|}{ Age (years) } \\
\hline $15-24$ & $126 / 69.6$ & $55 / 30.4$ & \multirow[t]{3}{*}{$2.6544 / 0.103$} & $83 / 45.9$ & $98 / 54.1$ & \multirow[t]{3}{*}{$0.394 / 0.530$} \\
\hline$\geq 25$ & $110 / 61.5$ & $69 / 38.5$ & & $88 / 49.2$ & $91 / 50.8$ & \\
\hline Total & $236 / 65.6$ & $124 / 34.4$ & & $171 / 47.5$ & $189 / 52.5$ & \\
\hline \multirow{2}{*}{ Variables } & \multicolumn{2}{|c|}{ Cyproheptadine tablet } & \multirow{2}{*}{ Khi2/p } & \multicolumn{2}{|c|}{ Diclofenac injectable } & \multirow{2}{*}{ Khi2/p } \\
\hline & Users & Non users & & users & Non users & \\
\hline \multicolumn{7}{|l|}{ Age (years) } \\
\hline $15-24$ & $141 / 77.9$ & $40 / 22.1$ & \multirow{3}{*}{$24.569 /<0.001$} & $81 / 44.8$ & $100 / 55.2$ & \multirow{3}{*}{$0.427 / 0.513$} \\
\hline$\geq 25$ & $95 / 53.1$ & $84 / 46.9$ & & $74 / 41.3$ & $105 / 58.7$ & \\
\hline Total & $236 / 65.6$ & $124 / 34.4$ & & $155 / 43.1$ & $205 / 56.9$ & \\
\hline
\end{tabular}

\subsection{Regarding the Route of Administration}

As shown in Table 4, Tetracycline capsule, Dexamethasone and Cyproheptadine tablets, were sometimes administered via anal route at the rate of $36 \%, 30.1 \%$ and $38.1 \%$ respectively and Diclofenc injectable which is more and more used by topical application in $89.7 \%$ of self-medication cases, that is to say on the head to relieve the pain of braids.

Table 4. Mode of administration

\begin{tabular}{|c|c|c|c|c|}
\hline \multirow{2}{*}{ Variable } & \multicolumn{2}{|c|}{ Dexamethasone tablet $n=236$} & \multicolumn{2}{|c|}{ Tetracycline capsule $n=203$} \\
\hline & Recommended & Non recommended & Recommended & Non recommended \\
\hline \multicolumn{5}{|c|}{ 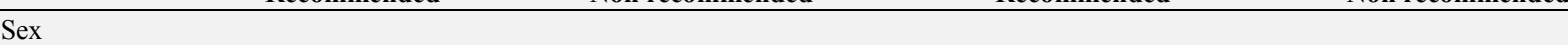 } \\
\hline Male & $61 / 100$ & $0 / 0$ & $70 / 61.4$ & $44 / 38.6$ \\
\hline Female & $104 / 59.4$ & $71 / 40.6$ & $60 / 67.4$ & $29 / 32.6$ \\
\hline Total & $165 / 69.9$ & $71 / 30.1$ & $130 / 64$ & $73 / 36$ \\
\hline \multicolumn{5}{|l|}{ Age } \\
\hline $15-24$ & $73 / 57.9$ & $53 / 42.1$ & $61 / 53.5$ & $53 / 46.5$ \\
\hline$\geq 25$ & $92 / 83.6$ & $18 / 16.4$ & $69 / 77.5$ & $20 / 22.5$ \\
\hline \multirow{2}{*}{ Variable } & \multicolumn{2}{|c|}{ Cyproheptadine tablet $\mathrm{n}=236$} & \multicolumn{2}{|c|}{ Diclofenac injectable $n=155$} \\
\hline & Recommended & Non recommended & Recommended & Non recommended \\
\hline \multicolumn{5}{|c|}{ (2) } \\
\hline Male & $66 / 100$ & $0 / 0$ & $6 / 100$ & $0 / 0$ \\
\hline Female & $80 / 47.1$ & $90 / 52.9$ & $10 / 6.7$ & $139 / 93.3$ \\
\hline Total & $146 / 61.9$ & $90 / 38.1$ & $16 / 10.3$ & $139 / 89.7$ \\
\hline \multicolumn{5}{|l|}{ Age } \\
\hline $15-24$ & $70 / 49.6$ & $71 / 50.4$ & $6 / 7.4$ & $75 / 92.6$ \\
\hline$\geq 25$ & $76 / 80$ & $19 / 20$ & $10 / 13.5$ & $64 / 86.5$ \\
\hline
\end{tabular}

\subsection{Dosage Used}

Medications contain specific amounts of pharmaceutically active ingredients, which significantly determine the duration and effectiveness of treatment. Thus, the respect of the dosage of the drugs as recommended by the doctor is more than necessary. One of the significant disadvantages of selfmedication is the non-compliance with the dosage. This has been noted for the products discussed in this report. It can be noted that dosages of Amoxycillin capsule, ECP and paracetamol tablets are respected in $88.9 \%, 100 \%$ and $90.1 \%$ of cases, respectively. However, this is not the case for Dexamethasone (80.1\%), Cyproheptadine (86\%), Levamisole (75.9\%), Albendazole tablets (76.1\%), Tetracycline capsule $(68.9 \%)$, Quinine $(67.8 \%)$ and Diclofenac injectables $(94.8 \%)$, where the doses are often increased to have quick 
effect (Table 5).

Table 5. Dosage used.

\begin{tabular}{|c|c|c|c|c|c|}
\hline Variable & Recommended & Non recommended & Variable & Recommended & Non recommended \\
\hline \multicolumn{6}{|c|}{ Dexamethasone tablet $n=236$} \\
\hline Sex & & & Age & & \\
\hline Male & $41 / 67.2$ & $20 / 32.8$ & $15-24$ & $26 / 20.6$ & $100 / 79.4$ \\
\hline Female & $6 / 3.4$ & $169 / 96.6$ & $\geq 25$ & $21 / 21$ & $89 / 89$ \\
\hline Total & $47 / 19.9$ & $189 / 80.1$ & Total & $47 / 19.9$ & $189 / 80.1$ \\
\hline \multicolumn{6}{|c|}{ Cyproheptadine tablet $n=236$} \\
\hline Male & $23 / 34.8$ & $43 / 65.2$ & $15-24$ & $21 / 14.9$ & $120 / 85.1$ \\
\hline Female & $10 / 5.9$ & $160 / 94.1$ & $\geq 25$ & $12 / 12.6$ & $83 / 87.4$ \\
\hline Total & $33 / 14$ & $203 / 86$ & Total & $33 / 14$ & $203 / 86$ \\
\hline \multicolumn{6}{|c|}{ Levamisole tablet $n=191$} \\
\hline Male & $20 / 76.9$ & $6 / 23.1$ & $15-24$ & $15 / 14.9$ & $86 / 85.1$ \\
\hline Female & $26 / 15.8$ & $139 / 84.2$ & $\geq 25$ & $31 / 34.4$ & $59 / 65.6$ \\
\hline Total & $46 / 24.1$ & $145 / 75.9$ & Total & $46 / 24.1$ & $145 / 75.9$ \\
\hline \multicolumn{6}{|c|}{ Albendazole tablet $\mathrm{n}=180$} \\
\hline Male & $30 / 78.9$ & $8 / 21.1$ & $15-24$ & $25 / 23.8$ & $80 / 76.2$ \\
\hline Female & $13 / 9.2$ & $129 / 90.8$ & $\geq 25$ & $18 / 24$ & $57 / 76$ \\
\hline Total & $43 / 23.9$ & $137 / 76.1$ & Total & $43 / 23.9$ & $137 / 76.1$ \\
\hline \multicolumn{6}{|c|}{ Quinine injectable $n=171$} \\
\hline Male & $8 / 100$ & $0 / 0$ & $15-24$ & $25 / 30.1$ & $58 / 69.9$ \\
\hline Female & $47 / 28.9$ & $116 / 71.1$ & $\geq 25$ & $30 / 34.1$ & $58 / 65.9$ \\
\hline Total & $55 / 32.2$ & $116 / 67.8$ & Total & $55 / 32.2$ & $116 / 67.8$ \\
\hline \multicolumn{6}{|c|}{ Diclofenac injectable $n=155$} \\
\hline Male & $6 / 100$ & $0 / 0$ & $15-24$ & $5 / 6.2$ & $76 / 93.8$ \\
\hline Female & $2 / 1.3$ & $147 / 98.7$ & $\geq 25$ & $3 / 4.1$ & $71 / 95.9$ \\
\hline Total & $8 / 5.2$ & $147 / 94.8$ & Total & $8 / 5.2$ & $147 / 94.8$ \\
\hline
\end{tabular}

Table 6. Reasons of use.

\begin{tabular}{|c|c|c|c|c|c|}
\hline \multicolumn{6}{|c|}{ Medications used } \\
\hline Variable & Rec. & Non rec. & Variable & Rec. & Non rec. \\
\hline \multicolumn{6}{|c|}{ Dexamethasone tablet $n=236$} \\
\hline Sex & & & Age & & \\
\hline Male & $21 / 34.4$ & $40 / 64.6$ & $15-24$ & $6 / 4.8$ & $120 / 95.2$ \\
\hline Female & $0 /$ & $175 / 100$ & $\geq 25$ & $15 / 13.6$ & $95 / 86.4$ \\
\hline Total & $21 / 8.9$ & $215 / 91.1$ & Total & $21 / 8.9$ & $215 / 91.1$ \\
\hline \multicolumn{6}{|c|}{ Cyproheptadine tablet $\mathrm{n}=236$} \\
\hline Male & $20 / 30.3$ & $46 / 69.7$ & $15-24$ & $11 / 7.8$ & $130 / 92.2$ \\
\hline Female & $6 / 3.5$ & $164 / 96.5$ & $\geq 25$ & $15 / 15.8$ & $80 / 84.2$ \\
\hline Total & 11 月 26 日 & $210 / 89$ & Total & 11 月 26 日 & $210 / 89$ \\
\hline \multicolumn{6}{|c|}{ Tetracycline capsule $n=203$} \\
\hline Male & $2 / 1.8$ & $112 / 98.2$ & $15-24$ & $12 / 14.8$ & $69 / 85.2$ \\
\hline Female & $10 / 11.2$ & $79 / 88.8$ & $\geq 25$ & $18 / 14.8$ & $104 / 85.2$ \\
\hline Total & $12 / 5.9$ & 191/94.1 & Total & $30 / 14.8$ & $173 / 85.2$ \\
\hline \multicolumn{6}{|c|}{ Levamizole tablet $n=191$} \\
\hline Male & $26 / 100$ & $0 / 0$ & $15-24$ & $35 / 34.6$ & $66 / 65.4$ \\
\hline Female & $31 / 18.8$ & $134 / 81.2$ & $\geq 25$ & $22 / 24.4$ & $68 / 75.6$ \\
\hline Total & $57 / 29.8$ & $134 / 70.2$ & Total & $57 / 29.8$ & $134 / 70.2$ \\
\hline \multicolumn{6}{|c|}{ Albendazole tablet $\mathrm{n}=180$} \\
\hline Male & $38 / 100$ & $0 / 0$ & $15-24$ & $35 / 33.3$ & $70 / 66.7$ \\
\hline Female & $11 / 7.7$ & $131 / 92.3$ & $\geq 25$ & $14 / 18.7$ & $61 / 81.3$ \\
\hline Total & $49 / 27.2$ & $131 / 72.8$ & Total & $49 / 27.2$ & $131 / 72.8$ \\
\hline \multicolumn{6}{|c|}{ Quinine injectable $n=171$} \\
\hline Male & $8 / 100$ & $0 / 0$ & $15-24$ & $16 / 19.3$ & $67 / 80.7$ \\
\hline Female & $26 / 16$ & $137 / 84$ & $\geq 25$ & $18 / 20.5$ & $70 / 79.5$ \\
\hline Total & $34 / 19.9$ & $137 / 80.1$ & Total & $34 / 19.9$ & $137 / 80.1$ \\
\hline \multicolumn{6}{|c|}{ Diclofenac injectable $n=155$} \\
\hline Male & $6 / 100$ & $0 / 0$ & $15-24$ & $5 / 6.2$ & $76 / 93.8$ \\
\hline Female & $2 / 1.3$ & $147 / 98.7$ & $\geq 25$ & $3 / 4.1$ & $71 / 95.9$ \\
\hline Total & $8 / 5.2$ & $147 / 94.8$ & Total & $8 / 5.2$ & $147 / 94.8$ \\
\hline
\end{tabular}




\subsection{Reasons of Use}

Since medications are not common consumers items, they should only be used for specific reasons and under the supervision of a physician. Unfortunately, in case of selfmedication, often other motivations are involved. As shown in Table 6, Dexamethasone and Cyproheptadine tablets are more exploited for their side effect in order to gain weight in
$91.1 \%$ and $89 \%$ of self-medication cases, respectively. Levamisole, Albendazole tablets and Quinine injectables are used as abortifacient in $70.2 \%, 72.8 \%$ and $80.1 \%$ of selfmedication cases, respectively. Tetracycline capsule is used as antidiarrheal and anti-hemorrhoidal in $94.1 \%$ of selfmedication cases. And Diclofenac injectable is used in $94.8 \%$ of cases on the head to relieve the pain of braids.

\subsection{Sources of Information}

Table 7. Patients source of information.

\begin{tabular}{|c|c|c|c|c|c|c|}
\hline Drugs used & $\operatorname{Sex} / \mathbf{n}$ & $\begin{array}{l}\text { Hawker n } \\
(\%)\end{array}$ & $\begin{array}{l}\text { Pharmacist or other } \\
\text { staff } n(\%)\end{array}$ & $\begin{array}{l}\text { Previous } \\
\text { experience n (\%) }\end{array}$ & $\begin{array}{l}\text { Family and/or } \\
\text { friends } n(\%)\end{array}$ & $\begin{array}{l}\text { Others sources } \\
\text { n (\%) }\end{array}$ \\
\hline \multirow{2}{*}{ Amoxicilline capsules } & $\mathrm{M} / 180$ & $3 / 1.7$ & $67 / 37.2$ & $80 / 44.4$ & $30 / 16.7$ & $0 / 0$ \\
\hline & $\mathrm{F} / 146$ & $0 / 0$ & $30 / 20.5$ & $60 / 41.1$ & $53 / 36.3$ & $3 / 2.1$ \\
\hline \multirow{2}{*}{ Emergency pill tablet } & $\mathrm{M} / 0$ & - & - & - & - & - \\
\hline & F180 & $17 / 9.5$ & $20 / 11.1$ & $2 / 1.1$ & $109 / 60.5$ & $32 / 17.8$ \\
\hline \multirow{2}{*}{ Paracétamol tablet } & $\mathrm{M} / 170$ & $4 / 2.3$ & $23 / 13.5$ & $30 / 17.6$ & $113 / 66.5$ & $0 / 0$ \\
\hline & $\mathrm{F} / 133$ & $0 / 0$ & $20 / 15$ & $13 / 9.8$ & $84 / 63.2$ & $16 / 12$ \\
\hline Dexamethasone tablet & F175 & $30 / 17.1$ & $5 / 2.9$ & $26 / 14.9$ & $100 / 57.1$ & $14 / 8$ \\
\hline \multirow{2}{*}{ Cyproheptadine tablet } & $\mathrm{M} / 66$ & $6 / 9.1$ & $6 / 9.1$ & $20 / 30.3$ & $20 / 30.3$ & $14 / 21.2$ \\
\hline & $\mathrm{F} / 170$ & $2 / 1.2$ & $2 / 1.2$ & $22 / 12.9$ & $133 / 78.2$ & $11 / 6.5$ \\
\hline \multirow{2}{*}{ Tetracycline capsule } & $\mathrm{M} / 114$ & $5 / 4.4$ & $20 / 17.5$ & $10 / 8.8$ & $60 / 52.6$ & $19 / 16.7$ \\
\hline & $\mathrm{F} / 89$ & $3 / 3.4$ & $41 / 46.1$ & $6 / 6.7$ & $25 / 28.1$ & $14 / 15.7$ \\
\hline \multirow{2}{*}{ Levamisole } & $\mathrm{M} / 26$ & $10 / 38.5$ & $2 / 7.7$ & $10 / 38.5$ & $4 / 15.4$ & $0 / 0$ \\
\hline & $\mathrm{F} / 165$ & $8 / 4.9$ & $3 / 1.8$ & $19 / 11.5$ & $131 / 79.4$ & $4 / 2.4$ \\
\hline Abendazole tablet & $\mathrm{F} / 142$ & $10 / 7$ & $5 / 3.5$ & $15 / 10.6$ & $107 / 75.4$ & $5 / 3.5$ \\
\hline \multirow{2}{*}{ Quinine injectable } & $\mathrm{M} / 8$ & $2 / 25$ & $2 / 25$ & $2 / 25$ & $2 / 25$ & $0 / 0$ \\
\hline & $\mathrm{F} / 163$ & $4 / 2.4$ & $4 / 2.4$ & $26 / 16$ & $108 / 66.3$ & $21 / 12.9$ \\
\hline \multirow{2}{*}{ Diclofenac injectable } & $\mathrm{M} / 6$ & $2 / 33.3$ & $2 / 33.3$ & $0 / 0$ & $2 / 33.3$ & $0 / 0$ \\
\hline & $\mathrm{F} / 149$ & $9 / 6$ & $6 / 4$ & $8 / 5.4$ & $115 / 77.2$ & $11 / 7.4$ \\
\hline
\end{tabular}

Several sources of information are involved regarding selfmedication, depending on the type of drug used. The five sources were mainly evaluated, namely: the hawker or marketing agents, previous experience, family members and/or friends, the pharmacist or his auxiliary and other sources. With regard to Amoxicillin capsule, the main source was the previous experience $(42.9 \%)$ while for all other products the main source was family and/or friends, ECP (60.5\%), Paracetamol (65\%), Dexamethasone (50.9\%), Cyprohetadine (64.9\%), Tetracycline (41.8\%), Levamisole (70.7\%), Albendasole (72.7\%), Quinine (64.3\%) and Diclofenac injectables (75.5\%), See table 7.

\section{Discussion}

This study allowed us to establish a list of ten mecidines mainly involved in self-medication practice among youth in Kinshasa. The following sections briefly describe each of the reported 10 medicines in terms of Amoxicillin and tetracyclin capsules, ECP, Paracetamol, Dexamethasone, Cyproheptadine, Levamisole and Albendazole tablets, Quinine and Diclofenac injetctables.

\subsection{Amoxicillin and Tatracycline Capsules}

Antibiotics, also konwn as antibacterials, can be defined as medications that destroy (bactericide) or slow down (bacteriostatic) the growth of bacteria. The risk linked to the phenomenon of resistance is becoming more and more widespread following the misuse of antibiotics, especially in developing countries where regulations concerning the use of medicines are not respected [2, 7]. As part of our study, $88.3 \%$ and $48.3 \%$ of pharmacists or their auxiliairies enroled in this study cited amoxicillin and tetracyclin capsules respectively among the 10 drugs often used in self-medication, which confirms the findings from the previous studies conducted in DRC and Nigeria $[8,9]$.

A study conducted in Eastern DRC [2], although among a particular population (students), yielded similar results: amoxicillin $(65.9 \%)$ and tetracycline $(21.5 \%)$. The somewhat high percentage in this study for both drugs can be explained by the fact that our study has assessed this practice in the population in all its entirety without distinction of social classes, probably involving people who have no information about the dangers of the drug misuse. Another similar study was conducted in Uganda [10], where amoxicillin and tetracycline capsules were once again among the most widely used antibiotics. Our results are slightly lower than those found in Uganda. This can be explained by the fact that in the previous study, the authors discussed the use of antibiotics in the context of specific infectious diseases, patients were 
obliged to use an antibiotic of their choice.

All these studies consistently demonstrated that population is highly exposed to antibiotic side effects. Thus, health authorities must do their utmost to regulate the use of drugs because antibiotic resistance is a permanent threat to public health worldwide [11].

\subsection{Paracetamol Tablet}

Paracetamol is the most widely used analgesic and antipyretic drug in the world. It can be obtained easily and without a prescription from a pharmacy. Given its long-term safe use many people do not fear possible negative effects with paracetamol [12]. Paracetamol was one of the most commonly used medications by self-medication in this study $(69.2 \%)$, which is similar to findings from the literature [13]. Currently, several studies are questioning prolonged use of paracetamol due to possible serious side effects on the cardiovascular and renal systems [14]. Even if paracetamol remains the most widely used over-the-counter and prescribed analgesic worldwide, measures of supervision must be taken to ensure the population safety.

\subsection{Levamisole Tablet}

Levamisole, which is a drug normally indicated against roundworms, is commonly used among youth in Kinshasa as a contraceptive in $70.2 \%$ of self-medication cases; it is used after unprotected intercourse to prevent an unwanted pregnancy $[5,6]$. The mechanism of action used for this drug is not known but its use is motivated by the fact that this drug is contraindicated in pregnancy, leading girls to believe that this drug can be used as an abortifacient. In $75.9 \%$ of selfmedication cases, the doses used are higher than the usual doses, increasing by this way the health risk of intoxication $[5,15]$.

\subsection{Emergency Contraceptif Pill Tablet}

ECP can be define as any drug or device that is used after unprotected sex to prevent pregnancy. Several studies published on the ECP raised concerns about the irrational use of this drug, especially among young ladies who can now freely engage in unprotected sexual intercourse, increasing the risk of contamination of sexually transmitted infectious diseases. Unlike previous studies, where many barriers did not facilitate access to the ECP [16], more and more youth and adolescents are aware of the existence and the use of this pill, as shown in our study (Table 3). In DRC, ECP can be purchased over the counter at pharmacies without prescription or proof of age, which is one of the reasons why the youth and adolescents are misusing ECP [5, 17]. Regulatory autorities need to pay attention to this growing interest of youth and adolescent on ECP to avoid long-term public health problem.

\subsection{Albendazole Tablet}

Albendazole is an anthelmintic medication. Same a Levamisole, Albendazole is used to treat infections caused by worms such as tapeworm. As shown in this survey, in $72.8 \%$ of self-medication cases women use albendazole tablet as abortive. They believe that albendazole tablet is effective in killing sperm and thus can prevent pregnancy. Because albendazole is not recommended during pregnancy, adolescents in Kinshasa are using this medication improperly or without the advice of a physician as contraceptive by increasing the recommended dosage in $76.1 \%$ of cases, exposing themselves to the risk of experiencing product side effects and toxicity $[5,6]$.

\subsection{Quinine Injectable}

Quinine is a medication used to treat malaria, a disease caused by parasites. False information about Quinine injectable believed to be contraceptive (but is not). It was found in this study that $68 \%$ of young women believe that increased Quinine dose is effective in killing sperm, consequently in $80.1 \%$ of self-medication cases young women in Kinshasa used quinine injectable after unprotected sex to prevent pregnancy. Similar results were reported in the previous studies in DRC $[5,6]$. Quinine is known as medication that has a low therapeutic index, which means an increased risk of dose-related adverse reactions. Thus, misusing Quinine can result in serious side effects (on the heart, kidneys, or blood cells) or death [18].

\subsection{Cyproheptadine Tablet}

Cyproheptadine is an antihistaminic used to ease allergy symptoms such as watery eyes, runny nose, itching eyes/nose, sneezing, hives and itching by blocking histaminic. It is an appetite stimulator with an additional anticholonergic, antiserotonergic, and local anesthetic properties [19].

As shown in this study, Cyproheptadine was one of the most used medicine $(55 \%)$ by self-medication. Among the cyproheptadine users, 59.7\% were aged between 15-24 year old. Similar results were reported in littérature [4], where cyproheptadine users aged between 18-26 year old, at the rates of $64.02 \%-90.24 \%$. It was also the case in the study by [3], where $59.6 \%$ of cyproheptadine users aged between $13-$ 24 year old. Ladies were more in cyproheptadine selfmedication $(72 \%)$, similar resultat $(88.9 \%)$ were recorded previously [3].

This study showed that in $38.1 \%$ of cases cyproheptadine was used by anal route, in $89 \%$ of cases cyproheptadine was used for cosmetic purpose and in $64.9 \%$ the main information source was family and/or friends. Similar results were reported in previous study [3], where in $40.9 \%$ of cases cyproheptadine was used by anal route, in $78 \%$ of cases cyproheptadine was used for cosmetic purpose and in $71.2 \%$ the main information source was family and/or friends. Cyproheptadine has many safety concerns linked to various side effects such as: sleepiness, dizziness, tremors irritability, blurred vision, nausea and diarrhea, liver toxicity and failure [20]. It was noted that in $86 \%$ of cases the dosage used by young women was higher than recommended, increasing by this way the population health 
problem. The regalatory body have to do their best to ensure the population safety.

\subsection{Dexamethasone Tablet}

Dexamethasone is a potent synthetic member of the glucocorticoid class of steroid drug. Dexamethasone is highly effective antiinflammatory, immunosuppressant and decongestant drugs. It is used in the treatment of many conditions, including rheumatic problems, a number of skin diseases, severe allergies, asthma, chronic obstructive lung disease, croup, brain swelling, and along with antibiotics in tuberculosis [19].

Dexamethasone was cited in $55 \%$ of self-medication cases by pharmacist or their auxiliairies in this study. Among the Dexamethasone users, $53.4 \%$ were aged between $15-24$ year old. Similar results were found in [4], where Dexamethasone users aged between 18-26 year old at rates of 64.02-90.24\%. As in reference [4], ladies were more in Dexamethasone selfmedication practice $(74.2 \%)$.

Our data demonstrated that in $30.1 \%$ of self-medication cases, youth people in Kinshasa used dexamethasone tablet by anal route, in $91.1 \%$ of self-mecication cases for cosmetic purpose and in $50.9 \%$ of self-medication cases the main information source was family and/or friends. Similar results were presented in [4], almost in $100 \%$ of cases, the cosmetic objective was involved. Although, authors did not discuss the route of administration, one of the cited cosmetic objective was to have buttock's protuberances. Regarding this objective, young women in Kinshasa believe that increasing the dosage and using the anal route for drug administration will result in quick effect. Unfortunately, Dexamethasone causes serious systemic side effects, specifically in high dose or chronic use, significantly decreases quality of life, life expectancy and increases health care costs [21]. Moreover, cyproheptadine and dexamethasone are over the counter medications, can be purchased without prescription in Kinshasa. Thus, the population is significantly misusing these two medicines as cosmetic agents for gaining weight. Most of time, the doses are increased and the route of administration modified, consequently increasing the public risk of becoming obese [22].

\subsection{Diclofenac Injectable}

Our study showed the use of injectable diclofenac topically, on the head by young girls in $96.1 \%$ of cases, in order to reduce the pain of braids by increasing the dosage used in $94.8 \%$ of self-medication cases. Further studies are required to find out the possible side effects associated with this misuse.

\section{Conclusion}

The aim of this study was to assess the extent to which self-medication is used and evaluate its risks among the youth population in DRC. The main findings of the present study include: (1) list of ten mecidines mainly involved in self-medication practice among youth in Kinshasa was established; (2) Two antibiotics are involved (amoxycillin and tetracycline); (3) females were more involved in nonprescription practice than males, most of time using side effects of the medicines; (4) sometime the mode of administration and the dosage of medicines are modified; (5) the main age group involved in self-medication practice range from 15 to 24 years old.

Possible solutions for preventing, or at least reducing, the dangerous consequences of irrational self-medication practice may include: (a) Use of appropriate educational programs to alert the community on the risk of misusing medication; (b) enforcement of existing laws regulating the pharmaceutical sector; (c) improving awareness of the general public and pharmacist or their auxilary about antibiotics and the risks associated with their abuse; (d) effective use of mass media like television, radio, newspaper and internet for improving population awareness about drug side effects and the dangerousness of self medication practice; (e) exposure of college and school students to the concept of ECP: Education on ECP will be an effective long term measure and (f) mass campaigns like road shows to demonstrate the consequences of irrational self-medication practice.

The present study described the practice of self-medication and drug misuse among the young population in the capital city of DRC. The obtained findings provide evidence of drug abuse by young Congolese people. This encourages further surveys to interrogate a wider range of parameters to facilitate national assessment of the self-medication in DRC, enabling the govenmental decision makers to initiate and implement appropriate measures to address self-medication and drug misuse.

\section{References}

[1] El Ezz NFA, Ez-Elarab H. S. (2011). Knowledge, attitude and practice of medical students towards self-medication a tain shams Universit, Egypt. Journal of Preventive Medicine and Hygiene. 52 (4): 196-200.

[2] Bunduki G. K., Mumbere M., Mbahweka F. K. (2017). Assessment of antibiotic self-edication pattern among University students in Eastern of the Congo. Journal of Pharmaceutical Research International 18 (1): 1-7. https://doi10.9734/JPRI/2017/31848.

[3] Lubelo A. M., Bavuidibo C. D., Mafuta E. M., Ndelo J. D., Mputu L. C. M., Kabundji D. M., Mutombo P. B. (2016). The misuse of cyproheptadine: a non-communicable disease risk behaviour in Kinshasa population, Democratic Republic of Congo. Substance abuse treatment, prevention and policy 11: 1-7. doi: 10.1186/s13011-016-0051-8.

[4] Mputu L. C. M., Ndelo M. P., Nuapia B. Y., Mwelo N. J. A, Pandi B. D., Sita Z. A., Ndelo J. D. (2017). Socio-cultural behavior camparative study of the misuse of cyproheptadine combined with dexamethasone in population, Democratic Republic of Congo. International Journal of Medicine Research. 2 (4): 17-22. 
[5] Hernandez J. H., Mbadu M., Garcia M., Matawa G. (2017). Awareness and perceptions of emergency contraceptive pills among women in Kinshasa, Democratic Republic of the Congo. International Perspectives on Sexual and Reproductive Health 43 (3): 121-130. https://Doi:10.1363/43 4417.

[6] Muanda M., Ndongo P. G., Taub L. M., Bertrand J. T. (2016) Barries to modern contraceptive use in Kinshasa, DRC. PLolS ONE. 11 (12): 1-13. https://doi10.137/journal.one.0167560.

[7] Rather I. A, Kim B. C et Al. (2017). Self-medication and antibiotic resistance: Crisis, current challenges, and prevention. Saudi Journal of Biological Sciences 24 (4): 808-812. https://doi10.1016/j.sjbs.2017.01.004.

[8] Kamala T., Katuala Y. et al. (2013). Antibiotic prescribing in DR Congo: a knowledge, attitude and practice survey among medical doctors and students. PLoS ONE 8 (2): e55495. https://doi10.1371/journal.pone.0055495. Epub2013 Feb 18.

[9] Ajibola O., Olusola Akintoye Omisakin O. A., Eze A. A., Omoleke S. A. (2018). Self-medication with antibiotics, attitude and knowledge of antibiotic resistance among community residents and undergraduate students in Northwest Nigeria. Diseases 2018; 6: 32-46. https://doi10.3390/diseases602003.

[10] Mbonye A. K., Buregyeya E., Rutebemberwa E., Clarke S. E., Lal S. et Al. (2016). Prescription for antibiotic at drug shops and strategies to improve quality of care and patient safety: a cross-sectional survey in the private sector in Uganda. British Medical Journal Open 6: e010632. doi: 10.1136/bmjopen2015-010632.

[11] O’Neill J. (2016). Tackling Drug-Resistant Infections Globally: Final Report and Recommendations. https://wellcomecollection.org/works/thvwsul.

[12] McCrae J. C., Morrison E. E. et al. (2018). Long-term adverse effects of paracetamol - a review. British Journal of Clininical $\begin{array}{llll}\text { pharmacology } & 84 & (10): & 2218-2230 .\end{array}$ https://doi10.1111/bcp.13656.

[13] PR. Shankar P. R., Partha P. Shenoy N. (2002). Selfmedication and non-doctor prescription practices in Pokhara valley, Western Nepal: a questionnaire-based study. BMC Family Practice. 3: 17. https://doi:10.1186/1471-2296-3-7.

[14] Roberts E, Delgado V et al. (2016). Paracetamol: not as safe as we thought? A systematic literature review of observational studies. Annals of the Rheumatic Diseases. 75 (3): 552-559. https://doi10.1136/annrheumdis-2014-206914. Epub 2015 Mar 2.

[15] Shin-Yu Lu, Tzu-Fan Chang, Chih-Jen Lin. (2019) Treatment effectveness of levamisole plus prednisolone on oral lichen planus patients with emphasis on levamisole-induced agranulocytosis or pancytopenia. Journal of Formosan Medical Association. 118: 1193-1201. https://doi/10.1016/j.fma.2019.03.007.

[16] Matungulu C. M. et al., (2015). Determinants of contraceptive use in Mumbunda health zone in Lubumbashi, DRC. Pan $\begin{array}{llll}\text { African Medical Journal. } 3 & \text { (22): } 329 .\end{array}$ https://doi10.11604/pamj.2015.22.329.6262.eCollection 2015.

[17] Julie H. Hernandez, Muanda Fidèle Mbadu, Melissa Garcia, Annie Glover (2018). The provision of emergency contraception in Kinshasa's private sector pharmacies: experiences of mystery clients. Contraception 97 (1): 57-61. https://doi10.1016/j.contraception.2017.08.001. Epub2017 Aug 10.

[18] Nsengi Y. Ntamabyaliro, Christian Burri et al. (2018). Drug use in the management of uncomplicated malaria in public health facilities in the DRC. Malaria Journal 17: 189-197. https://doi10.1186/s12936-018-2332-3.

[19] Bordag N., Klie S. et al. (2015). Glucocorticoid (dexamethasone) induced metabolome changes in healthy males suggest prediction of response and side effects. Scientific Reports 5: 15954. https://doi:10.1038/srep15954.

[20] Homnick D. N., Marks J. H., Hare K. L., Bonnema S. K. (2005). Longterm trial of cyproheptadine as an appetite stimulant in cystic fibrosis. Pediatric Pulmonology 40 (3): 251-6. https://doi10.1002/ppul.20265.

[21] Sarnes E. et al. (2011). Incidence and US costs of corticosteroid-associated adverse events: a systematic literature review. Clinical Therapeutics. 33 (10): 1413-32. https://doi10.1016/j.clinthera.2011.09.009. Epub 2011 Oct 13.

[22] Amar M. I., Adam Shama I. Y., Enaia A. A., Hind A. E. O., Hager A. M. (2013). Effects of various levels of oral doses dexamethasone Al-nagma abused as cosmetic by Sudanese women on Wistar rats. Journal of Medical Sciences 13 (6): 432-438. https://doi10.3923/jms.2013.432.438. 